\title{
Cirugía renal laparoscópica conservadora de parénquima. Experiencia inicial
}

\author{
Aguilera Bazán A, Alonso y Gregorio S, Pérez Utrilla M, Cansino Alcalde JR, Cisneros Ledo J, \\ De la Peña Barthel J.
}

Servicio de Urología. Hospital Universitario La Paz. Madrid.

Actas Urol Esp. 2007;31(5):477-481

\section{RESUMEN}

CIRUGÍA RENAL LAPAROSCÓPICA CONSERVADORA DE PARÉNQUIMA. EXPERIENCIA INICIAL

Presentamos nuestra experiencia inicial en cirugía renal laparoscópica conservadora de parénquima. Se trata de una cirugía exigente que requiere de una considerable experiencia en cirugía mínimamente invasiva. Esta técnica es particularmente atractiva comparada con la cirugía clásica abierta, debido a la incisión que precisa esta última y a la morbilidad que conlleva.

Palabras clave: Tumorectomía. Nefrectomía parcial. Laparoscopia. Cirugia conservadora de parénquima.

\section{ABSTRACT}

LAPAROSCOPIC NEPHRON SPARING SURGERY. INITIAL EXPERIENCE

We present our initial experience in laparoscopic nephron sparing surgery. It's a technically advanced procedure requiring considerable minimally invasive expertise. This technique is particularly attractive compared to an open conventional procedure with its larger incision and associated morbidity.

Keywords: Tumorectomy. Partial nephrectomy. Laparoscopic. Nephron sparing surgery.

$\mathrm{D}$ esde que se realizó la primera nefrectomía laparoscópica en 1991, la cirugía laparoscópica renal ha ido abarcando casos cada vez más complejos, y lo que inicialmente se trataba de una contraindicación para esta técnica, se ha ido convirtiendo a fuerza de adquirir mayor experiencia y desarrollo tecnológico en una clara alternativa a la cirugía abierta. Tal es el caso de la cirugía renal conservadora, que si bien no es la técnica ideal para iniciarse en laparoscopia, es cierto que cada vez son más las publicaciones que demuestran su buen resultado oncológico unido a las bondades de la cirugía mínimamente invasiva $^{1}$ (Figs. 1 y 2 ).

En este artículo presentamos la experiencia inicial de nuestro Servicio en cirugía renal conservadora laparoscópica a los 2 años de iniciarse el programa de laparoscopia renal.

\section{MATERIAL Y MÉTODOS}

Desde septiembre de 2004 hasta Septiembre de 2006 se han realizado en nuestro centro un total de 20 tumorectomías laparoscópicas por vía transperitoneal, 13 de ellos varones y 7 mujeres. En 12 casos la cirugia ha sido derecha y en 8 izquierda. En 10 ocasiones han sido tumores de polo inferior, 2 de polo superior y 8 de tercio medio. La edad media es de 54 años (37-72), el índice de masa corporal de $26 \mathrm{~kg} / \mathrm{m} 2$ (22-37), la valoración del riesgo anestésico es de 8 ASA1, 7 ASA2, 4 ASA3 y 1 ASA4. Del total de 20 casos, 6 presentan antecedentes de cirugía abdominal (30\%). Como contraindicación absoluta se ha considerado los pacientes monorrenos, tumores por encima de $4 \mathrm{~cm}$ o las localizaciones mesorrenales próximas al hilio. No se han descartado tumores de localización posterior o de polo superior. 


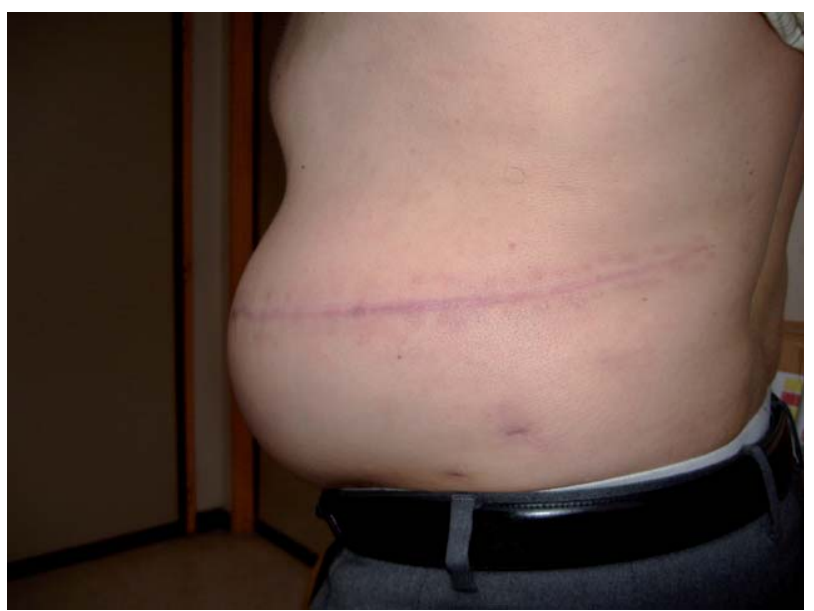

FIGURA 1

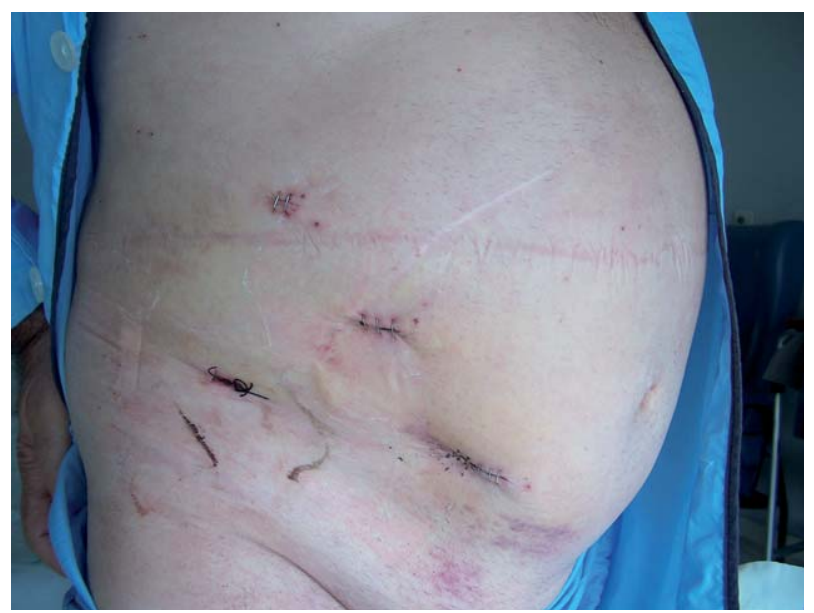

FIGURA 2

Como estudio preoperatorio principal se realiza una TAC con cortes transversales y coronales, lo que nos permite determinar la posición del tumor y su proximidad a la vía urinaria (Fig. 3).

La vía de abordaje elegida es la transperitoneal debido a las ventajas de espacio que supone. En aquellos casos en los que se sospeche que se pueda abrir la vía urinaria, se coloca un catéter ureteral previo al acto quirúrgico, que nos servirá para inyectar azul de metileno y asegurarnos de esta forma el correcto cierre de la vía. Desde un principio hemos optado por el clampaje del hilio renal, pero la técnica la hemos ido variando a medida que hemos adquirido experiencia, de manera que inicialmente realizábamos un clampaje con torniquete similar al descrito por el grupo de la Fundación Puigvert, con una disección com-

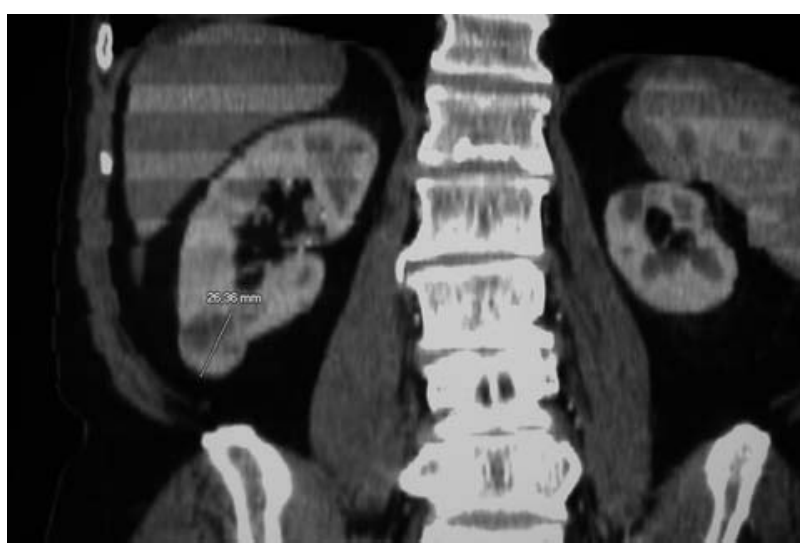

FIGURA 3

pleta de la arteria y vena renal ${ }^{2}$. Actualmente realizamos un clampaje en bloque del hilio renal con una pinza laparoscópica de Storz diseñada para ello teniendo cuidado de no abarcar también el uréter.

Tras marcar los límites de la resección con coagulación, realizamos la exéresis del tumor con corte frío de las tijeras, ya que el uso de coagulación en este punto puede enmascarar el plano tumoral. Tomamos biopsia del lecho tumoral que enviamos intraoperatoriamente para ser analizada por el patólogo. A continuación realizamos varias suturas contínuas con Vicryl 0 y un clip de material reabsorbible en los extremos (Lapr-Ty Ethicon), mientras inyectamos azul de metileno a través del catéter ureteral para identificar la vía urinaria abierta. Aplicamos un rollo de Surgicel y Flo Seal (Baxter) en el lecho tumoral y a continuación damos varios puntos de aproximación de parénquima con Vicryl 0. Finalmente declampamos el hilio renal y bajamos la presión del neumoperitoneo a $4 \mathrm{mmHg}$ para realizar hemostasia.

Todos los pacientes salen del quirófano con un tratamiento analgésico consistente en un infusor de $24 \mathrm{~h}$ de duración que contiene Tramadol 300 $\mathrm{mg}$, Ketorolako $90 \mathrm{mg}$ y Primperan $90 \mathrm{mg}$. Además se les deja pautado paracetamol $1 \mathrm{~g}$ cada 6 horas. La misma noche de la cirugía el paciente inicia tolerancia a líquidos.

\section{RESULTADOS}

No ha habido ningún caso de reconversión a cirugía abierta. El tiempo quirúrgico medio es de 143 minutos (90-240). El sangrado medio es de 293cc (50-1000), con 4 casos que han precisado 
transfusión (20\%). La estancia media hospitalaria es de 3,25 días (2-5). En 6 ocasiones ha sido necesaria la apertura de la vía urinaria (30\%). El tiempo de clampaje medio es de 21,5 min. Los resultados anatomopatológicos son los siguientes: 13 casos de adenocarcinoma de células claras pT1 de bajo grado, 2 pT3 de alto grado, 4 casos de oncocitoma, 1 adenoma renal. En cuanto a los márgenes de resección, hay dos casos que son positivos, uno de ellos se ha realizado una nefrectomía radical inmediatamente después ya que el resultado de la tumorectomía resultó ser un adenocarcinoma de células claras pT3 de alto grado y el otro caso, un tumor pT1 de bajo grado, se decidió seguimiento. El tamaño tumoral medio es de $3,0 \mathrm{~cm}(1,5-5,5)$.

En el apartado de complicaciones, todas las cirugias se han finalizado por laparoscopia. Hemos tenido dos casos de sangrado intraoperatorio de 1000 cc por clampaje inadecuado del hilio renal. Otros dos casos precisaron transfusión en el postoperatorio inmediato. Ha habido un caso de insuficiencia renal manejada médicamente (el tiempo de isquemia caliente fue de 30 min.), 1 caso de urinoma infectado, como complicación diferida, que precisó drenaje percutáneo. En el paciente número 11, el catéter ureteral se comportaba como obstructivo, motivo por el cual presentó episodios de dolor cólico en el postoperatorio inmediato. Tras comprobar con control radiológico el correcto cierre de la vía urinaria se le retiró el catéter.

\section{DISCUSIÓN}

La laparoscopia renal es ya una realidad como alternativa a la cirugía abierta, aunque parece que ésta última continúa siendo el gold Standard $^{3-6}$. Dentro de la cirugía renal laparoscópica, la cirugía conservadora de parénquima es todo un desafío que debemos acometer con la máxima experiencia laparoscópica posible y el material adecuado. En la actualidad contamos con series grandes y de suficiente seguimiento como para pensar que se trata de una técnica oncológicamente segura en manos experimentadas $^{1}$.

En nuestra experiencia inicial en esta técnica hemos decidido descartar tumores por encima de $4 \mathrm{~cm}$ debido al dudoso control oncológico de la cirugia parcial en estos tumores ${ }^{7}$, aunque en la actualidad pueda haber autores que se planteen la posibilidad de incrementar el tamaño tumoral con seguridad oncológica hasta $7 \mathrm{~cm}$ en pacientes con anatomía favorable ${ }^{8}$. Otras dos contraindicaciones que consideramos dependientes de la experiencia quirúrgica han sido la de tumores de localización próxima al hilio renal, debido a la dificultad en el control de sangrado y aquellos tumores localizados en pacientes monorrenos, en los que creemos de elección la tumorectomía por vía abierta con enfriamiento renal. Tumores de localización posterior o en polo superior no los hemos considerado como una contraindicación laparoscópica, ya que con una buena liberación del riñón se puede tener un acceso adecuado a estas posiciones (Figs. 4 y5).

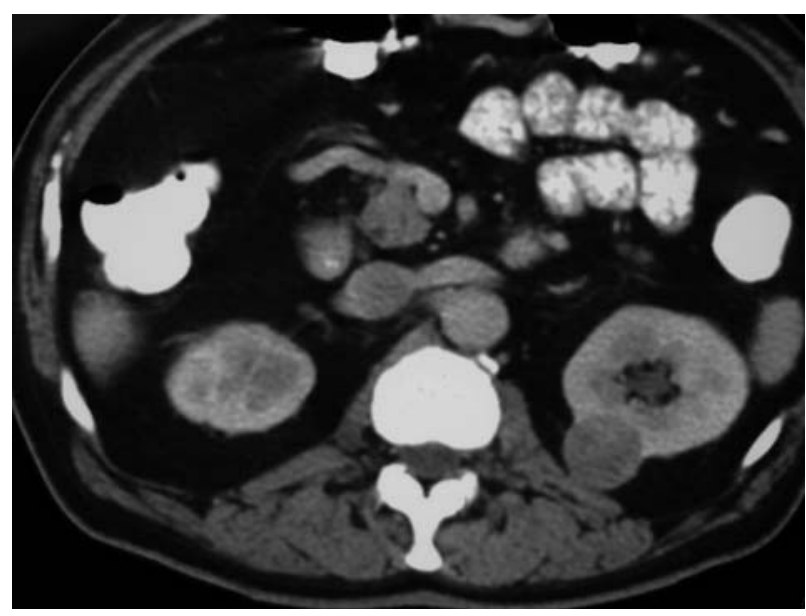

FIGURA 4

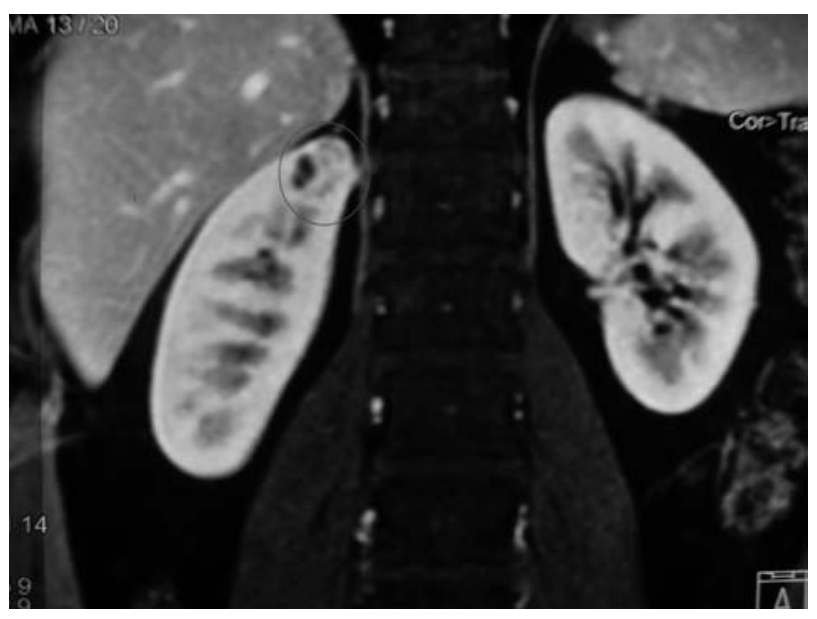

FIGURA 5 
En cuanto a la vía de abordaje, creemos que la transperitoneal tiene claras ventajas frente a la retroperitoneoscopia debido al mayor espacio de trabajo que se consigue, lo que repercute en la colocación de los puertos y por tanto en la mayor facilidad para la disección tumoral y sutura del lecho quirúrgico ${ }^{9,10}$. Incluso los tumores de cara posterior los hemos abordado por esta vía, con una mayor disección de todo el riñón para que nos permita voltearlo prácticamente por completo.

Decidimos desde un principio proceder al clampaje del hilio renal, si bien, inicialmente disecábamos la arteria y la vena para proceder al clampaje arterial con un torniquete, esta maniobra supone un tiempo mayor que el clampaje en bloque, y lo que es más importante, requiere de una disección mayor de la arteria y la vena, con el riesgo que conlleva de lesión vascular. Por estos motivos decidimos variar la técnica y usar una pinza laparoscópica para clampar la arteria y vena renal en bloque. En los primeros casos el resultado no fue el esperado ya que la pinza que empleábamos no tenía la suficiente fuerza para colapsar por completo la arteria, de manera que el sangrado fue importante (aproximadamente $1000 \mathrm{cc}$ ) en los primeros pacientes y el margen de disección del tumor era más incierto. Actualmente disponemos de una pinza de clampaje vascular que ejerce mayor presión, aunque debemos evitar disecar excesivamente la arteria, ya que se podría producir una lesión en la íntima del vaso. No creemos que una isquemia caliente en torno a los 30 min tenga una repercusión importante en la función renal, como ya se ha demostrado en diversos estudios ${ }^{11,12}$.

Preferimos realizar la sección del parénquima renal con corte frío de tijeras. En algunas ocasiones hemos empleado algún sistema de corte-coagulación utilizado por los cirujanos generales en las hepatectomías, pero no nos ha gustado ya que distinguimos peor el margen de parénquima renal sano que queremos respetar.

Una vez terminada la sección del parénquima renal, procedemos a la sutura sobre el lecho tumoral que debe ser lo más rápida posible, para ello empleamos el clip plástico que nos ahorra tiempo de isquemia caliente. El empleo de pegamento biológico tras la sutura nos parece funda- mental para reducir el sangrado y probablemente tenga un papel importante en el cierre de la vía urinaria $^{8,13}$.

De las complicaciones presentadas la tasa de sangrado es más elevada, 20\%, que en otras series en donde se encuentra en torno al 10\%1. El mal funcionamiento de la pinza de Satinski que usamos inicialmente ha sido un factor determinante en este aspecto. Los sangrados más aparatosos los hemos tenido durante la sección del parénquima por un mal clampaje del hilio, más que en el postoperatorio por problema de sutura del lecho tumoral. Una de las transfusiones realizada en el postoperatorio se trataba de un paciente con antecedentes de angor y una $\mathrm{Hb}$ de $9,8 \mathrm{mg} /$ dl tras la cirugía. La tasa de urinoma es la encontrada en otras series, en torno al 5\%. Sin duda, el empleo de azul de metileno ayuda a identificar la vía urinaria abierta $\mathrm{y}$, por otra parte, la colocación de un catéter ureteral doble J en estos pacientes tiene su importancia. La aplicación de pegamentos probablemente juegue un papel importante, aunque nuestra serie es todavía demasiado corta para poder analizar este aspecto.

El caso de insuficiencia renal presentado, se trataba de un paciente de 71 años con antecedentes cardiovasculares importantes (accidente cerebrovascular, claudicación intermitente, fibrilación auricular) y con un tiempo de isquemia caliente de 30 minutos. Se manejó médicamente, no precisando diálisis y siendo dado de alta con una creatinina normal.

La tasa de márgenes positivos debe reducirse a medida que se adquiere experiencia y el tamaño de la serie aumenta. En este sentido, que el clampaje sea adecuado es fundamental ya que nos permitirá ver claramente el plano de parénquima renal sano. Uno de los márgenes positivos fue tratado con nefrectomía radical laparoscópica posteriormente, debido al grado tumoral (pT3 alto grado). El otro caso se trata de un tumor pT1 de bajo grado y lleva un seguimiento de 24 meses libre de enfermedad. El seguimiento medio de toda la serie es de 12 meses, no habiéndose objetivado recidiva local o en los puertos de trabajo.

No ha habido complicaciones intestinales ni lesiones viscerales. Tampoco se han producido complicaciones de pared abdominal. 


\section{CONCLUSIONES}

La cirugía laparoscópica conservadora de parénquima es una técnica claramente exigente que se debe realizar cuando se tenga un manejo adecuado de la sutura intracorpórea. Es también de vital importancia un clampaje adecuado del hilio renal (torniquete o pinza vascular) ya que la complicación inmediata más temida (hemorragia incontrolable) y el resultado oncológico (márgenes positivos) tiene una relación directa con este hecho. La selección de pacientes con tumores más fácilmente accesibles (polo inferior, exofiticos) reducirá la posibilidad de complicaciones.

\section{REFERENCIAS}

1. Moinzadeh A, Gill S, Finelli A, Kaouk J, Desai M. Laparoscopic partial nephrectomy: 3-year followup. J Urol. 2006 Feb;175(2):459-462.

2. Rosales A, Salvador J,. De Graeve N, Angerri O, Villavicencio H. Clamping of the renal artery in laparoscopic partial nephrectomy: an old device for a new technique. Eur Urol. 2005 Jan;47(1):98-101.

3. Ono Y, Hattori R, Gotoh M, Yoshino Y, Yoshikawa Y, Kamihira O. Laparoscopic radical nephrectomy for renal cell carcinoma: the standard of care already?. Curr Opin Urol. 2005 Mar; 15(2):75-78.

4. Chan DY, Cadeddu JA, Jarrett TW, Marshall FF, Kavoussi LR. Laparoscopic radical nephrectomy: cancer control for renal cell carcinoma. J Urol. 2001 Dec;166(6):2095-2099; discussion 2095-2100.

5. Portis AJ, Yan Y, Landman J, Chen C, Barrett PH, Fentie DD et al. Long-term follow up after laparoscopic radical nephrectomy. J Urol. 2002 Mar;167(3):1257-1262.
6. Dunn MD, Portis AJ, Shalhav AL, Arieh L, Elbahnasy, Heidorn $\mathrm{C}$ et al. Laparoscopic versus open radical nephrectomy: a 9-year experience. J J Urol. 2000 Oct;164(4):11531159.

7. Patard JJ, Shvarts O, Lam JS, Pantuck AJ, Kim HL, Ficarra $\mathrm{V}$ et al. Safety and efficacy of partial nephrectomy for all T1 tumors based on an international multicenter experience. J Urol. 2004 Jun;171(6 Pt 1):2181-2185, quiz 2435.

8. Haber GP, Gill IS. Laparoscopic Partial Nephrectomy: Contemporary Technique and Outcomes. Eur Urol. 2006 Apr;49(4):660-665. Epub 2006 Feb 20.

9. Desai MM, Strzempkowski B, Matin SF, Steinberg AP, Ng C, Meraney AM et al. Prospective randomized comparison of transperitoneal versus retroperitoneal laparoscopic radical nephretctomy. J Urol. 2005 Jan;173(1):38-41.

10. Abdelmaksoud A, Biyani CS, Bagheri F, Janetschek G. Laparoscopic approaches in urology. BJU Int. 2005 Feb; 95(2):244-249.

11. Nadu A, Kitrey N, Mor Y, Golomb J, Ramon J. Laparoscopic partial nephrectomy: is it advantageous and safe to clamp the renal artery?. Urology. 2005 Aug;66(2):279-282.

12. Desai MM, Gill IS, Ramani AP, Spaliviero M, Rybicki L, Kaouk JH. The impact of warm ischaemia on renal function after laparoscopic partial nephrectomy. BJU Int. 2005 Feb;95(3):377-383.

13. Ramani AP, Desai MM, Steinberg AP, Ng CS, Abreu SC, Kaouk JH et al. Complications of laparoscopic partial nephrectomy in 200 cases. J Urol. 2005 Jan;173(1):42-47.

Correspondencia autor: Dr. A. Aguilera Bazán

Servicio de Urología. Hospital La Paz

$\mathrm{P}^{\circ}$ de la Castellana 261. 28046 Madrid

Tel.: 917277000

Email autor: aaguilera11@yahoo.es

Información artículo: Original - Cáncer de riñón

Trabajo recibido: diciembre 2006

Trabajo aceptado: abril 2007 